\title{
Density Flow in Dynamical Networks via Mean-field Games
}

\author{
Dario Bauso and Xuan Zhang and Antonis Papachristodoulou
}

\begin{abstract}
Current distributed routing control algorithms for dynamic networks model networks using the time evolution of density at network edges, while the routing control algorithm ensures edge density to converge to a Wardrop equilibrium, which was characterized by an equal traffic density on all used paths. We rearrange the density model to recast the problem within the framework of mean-field games. In doing that, we illustrate an extended state-space solution approach and we study the stochastic case where the density evolution is driven by a Brownian motion. Further, we investigate the case where the density evolution is perturbed by a bounded adversarial disturbance. For both the stochastic and the worst-case scenarios, we provide conditions for the density to converge to a preassigned set. Moreover, we analyze such conditions from two different perspectives, repeated games with vector payoffs and inclusion theory.
\end{abstract}

\section{INTRODUCTION}
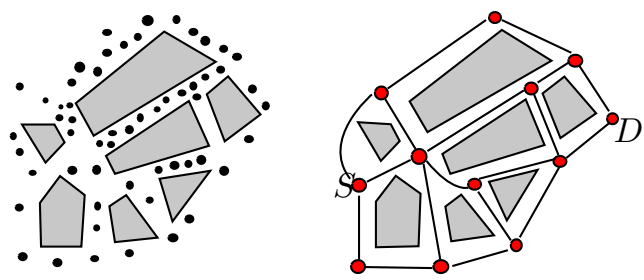

Fig. 1: Pedestrian flow modelled via network flow with a source node $S$ and a destination node $D$.

In this paper we study a routing problem over a network. The problem setup involves a population of individuals or players traversing the edges of a network in an attempt to reach a destination node starting from a source node (see, e.g., Fig. 1). From a microscopic standpoint, each player jumps from one edge to an adjacent one according to a continuoustime Markov model. Players select the transition rates, which represent the control. From a macroscopic perspective, each edge is characterized by dynamics describing the time evolution of the density of players on that edge. These dynamics

The preliminary conference version of this work was presented in CDC 2014 [8]. A short version of this paper appears in [4, Chapter 18]. The work of D. Bauso has been supported by the PRIN 20103S5RN3 "Robust decision making in markets and organization".

Dario Bauso is with the Department of Automatic Control and Systems Engineering, The University of Sheffield, Mappin Street Sheffield, S1 3JD, United Kingdom, and with the Dipartimento di Ingegneria Chimica, Gestionale, Informatica, Meccanica, Università di Palermo, V.le delle Scienze, 90128 Palermo, Italy. D. Bauso has done part of this work while he was academic visitor at the Department of Engineering Science, University of Oxford, UK. \{d.bauso@sheffield.ac.uk\}

$\mathrm{X}$. Zhang is with the School of Engineering and Applied Sciences, Harvard University, 29 Oxford Street, Cambridge, MA 02138, USA \{xuan_zhanglg.harvard.edu\}

Antonis Papachristodoulou is with Department of Engineering Science, University of Oxford, Parks Road, Oxford, OX1 3PJ, UK \{antonis@eng.ox.ac.uk\} take the form of a classical forward Kolmogorov Ordinary Differential Equation (ODE). We then extend our analysis to the case where the Kolmogorov equation turns into a Stochastic Differential Equation (SDE) driven by a Brownian motion. Finally, in the last part of this paper, we investigate the case where the Kolmogorov Ordinary Differential Equation is subject to adversarial inputs.

\section{A. Related literature}

Previous work considered distributed routing problems using a different setup [14], [15]. In this paper, we provide a detailed analysis of a related problem via mean-field games theory. The theory on mean-field games originated in the work of M. Y. Huang, P. E. Caines and R. Malhamé [19], [20], [21] and independently in that of J. M. Lasry and P. L. Lions [23], [24], [25], where the now standard terminology of Mean-Field Games (MFG) was introduced. In addition to this, the closely related notion of Oblivious Equilibria for large population dynamic games was introduced by G. Weintraub, C. Benkard and B. Van Roy [34] in the framework of Markov Decision Processes.

The problem we analyze in this paper has striking similarities to the optimal planning problem [1], [9], [27], [29] which in turn can be linked back to mean-field games. Essentially, in optimal planning problems the idea is to drive the density of players from a given initial configuration to a target one in a given time by an appropriate design of the (optimal) decisions of the agents.

Mean-field games arise in several application domains such as economics, physics, biology, and network engineering (see [1], [2], [18], [21], [28], [30], [33]). Finite state mean-field games have been discussed in [16], [17]. Explicit solutions in terms of mean-field equilibria are not common unless the problem has a linear quadratic structure [3]. In this sense, a variety of solution schemes have been recently proposed based on discretization and/or numerical approximations [1]. Meanfield games have precursors in anonymous games and aggregative games building upon the notion of mass interaction and can be seen as a stationary mean-field game in dynamic discrete time [22]. More recently, robustness notions have been introduced in mean-field games. Robust mean-field games aim to achieve robust performance or stability in the presence of unknown disturbances when there is a large number of players. Their relationship with risk-sensitive games and risk-neutral games has been analyzed in [31], [32].

\section{B. Main contribution}

The main contributions of this paper can be summarized as follows. First, we provide a mean-field game formulation of the problem. In doing this we analyze which aspects can help 
turning the mean-field game into a deterministic control problem. Second, we illustrate an extended state-space solution approach. Such a solution approach uses nonstandard meanfield techniques and is an alternative method to classical fixedpoint methods. Third, we study the stochastic case where the density evolution is driven by a Brownian motion. Additional results, that make this paper different from its conference version [8], are the following. We investigate the case where the density evolution is perturbed by a bounded adversarial disturbance. For this case, we provide two convergence conditions that are proven to be equivalent. We also show that convergence is guaranteed under certain assumptions that were first established in the context of repeated games with vector payoffs [6], [26]. On the other hand, the second condition takes the form of set inclusion and allows us to link the results to well-known convergence conditions in robust dynamic flow networks already available in the literature [5], [12], [13].

\section{Structure of the paper}

The rest of the paper is organized as follows. In Section II we illustrate the problem and introduce the model. In Section III we present the main results of the paper. In Section IV we provide numerical examples. Finally, in Section V we draw some conclusions.

\section{MODEL AND PROBLEM SET-UP}

Let a graph $G=(V, E)$ be given where $V=\{1, \cdots, n\}$ is the set of vertices and $E=\{1, \cdots, m\}$ the set of edges. Let us denote by $\varepsilon^{+}(i)$ and $\varepsilon^{-}(i)$ the sets of outgoing edges from $i$ and incoming edges to $i$ respectively, $\forall i \in V$. We consider a "large population" of individuals or players of which each one is characterized by a time-varying state $X(t) \in E$ at time $t \in[0, T]$, where $[0, T]$ is the time horizon window. The routing policy is described by a vector-valued function $\alpha(\cdot): \mathbb{R}_{+} \rightarrow[0,1]^{m}, t \mapsto \alpha(t)$ where $[0,1]^{m}$ denotes the $m$-dimensional column vector whose entries are within the interval $[0,1]$. Moreover, we have $\sum_{e \in \varepsilon^{+}(i)} \alpha_{e}=1$ where $\forall i \in V$ and $\alpha_{e}$ is the $e^{\text {th }}$ entry of $\alpha(t)$. In other words, $\alpha(t)$ lives in the space $\Delta^{\left|\varepsilon^{+}(1)\right|} \times \cdots \times \Delta^{\left|\varepsilon^{+}(n)\right|}$ where $\Delta^{\left|\varepsilon^{+}(i)\right|}$ denotes the simplex in $\mathbb{R}^{\left|\varepsilon^{+}(i)\right|}$ and $\left|\varepsilon^{+}(i)\right|$ is the cardinality of set $\varepsilon^{+}(i)$ (number of outgoing edges from $i$ ), $\forall i \in V$. Let $k \in E$ be the player's state. The state evolution of a single player is then captured by the following continuoustime Markov stochastic process:

$$
\begin{aligned}
& \{X(t), t \geq 0\}, \\
& q_{k j}\left(h, \phi_{k}, \alpha_{j}\right)= \begin{cases}\alpha_{j} \phi_{k} h & j \in \operatorname{Adj}(k), \\
1-\phi_{k} h, & j=k, \\
0, & \text { otherwise, }\end{cases}
\end{aligned}
$$

where $q_{k j}\left(h, \phi_{k}, \alpha_{j}\right)\left(q_{k j}\right)$ are the infinitesimal transition probabilities from $k$ to $j, h$ is the infinitesimal time interval, $\phi_{k} \in \mathbb{R}_{+}$is the transition rate in state $k \in E$, and $\operatorname{Adj}(k)=\left\{j \in E \mid j \in \varepsilon^{+}(i), k \in \varepsilon^{-}(i)\right\}$ represents the set of adjacent edges to $k$. In (1), from previous definitions of the control (the routing policy), we know that $\alpha$ belongs to the polytope $\mathcal{U}=\left\{\alpha \in[0,1]^{m} \mid\left\{\alpha_{j}\right\}_{j \in \varepsilon^{+}(i)} \in \Delta^{\left|\varepsilon^{+}(i)\right|}\right.$, for all $\left.i=1, \ldots, n\right\} . g(\cdot): E \times[0,1]^{m} \rightarrow[0,+\infty[,(x, \rho) \mapsto g(x, \rho)$ of the form
Essentially, the above is equivalent to saying $\sum_{j \in \varepsilon^{+}(i)} \alpha_{j}=1$ for all $i=1, \ldots, n$.

Denote by $\rho$ the vector of densities on edges, which means that the sum of the components is equal to one. Thus we have $\rho \in \mathcal{D}:=\left\{\hat{\rho} \in[0,1]^{m}: \sum_{e \in E} \hat{\rho}_{e}=1\right\}$, where $\hat{\rho}_{e}$ is the $e^{\text {th }}$ entry of $\hat{\rho}$. Let the flow function $f(\cdot): \mathcal{D} \rightarrow \mathbb{R}_{+}^{m}$, be given by $f_{e}(\rho)=\phi_{e} \rho_{e}$, where $f_{e}(\rho)$ is the $e^{\text {th }}$ entry of $f(\rho)$. The density evolution can be described by the Kolmogorov ODE given by

$$
\left\{\begin{array}{l}
\dot{\rho}(t)=\left(\tilde{B}^{T}(\alpha) \hat{B}-I\right) f(\rho) \\
\rho(0)=\rho_{0}
\end{array}\right.
$$

where

- the matrix-valued function $\tilde{B}(\cdot): \mathcal{U} \rightarrow[0,1]^{n \times m}$, $\alpha \mapsto \tilde{B}(\alpha)$, which relates nodes to outgoing edges, i.e., $\tilde{B}_{i j}(\alpha)=\alpha_{j}$ if $j \in \varepsilon^{+}(i)$ and $\tilde{B}_{i j}(\alpha)=0$ otherwise. Here $[0,1]^{n \times m}$ denotes the $n \times m$-dimensional matrix whose entries are within the interval $[0,1]$, and $\tilde{B}_{i j}(\alpha)$ is the entry in the $i^{\text {th }}$ row and $j^{\text {th }}$ column of $\tilde{B}(\alpha)$.

- the matrix $\hat{B} \in\{0,1\}^{n \times m}$ relates nodes to incoming edges, i.e., $\hat{B}_{i j}=1$ if $j \in \varepsilon^{-}(i)$ and $\hat{B}_{i j}=0$ otherwise. Here $\{0,1\}^{n \times m}$ denotes the $n \times m$-dimensional matrix whose entries are either 0 or 1 , and $\hat{B}_{i j}$ is the entry in the $i^{\text {th }}$ row and $j^{\text {th }}$ column of $\hat{B}$

- $\rho_{0}$ is the initial density and it is assigned.

Equation (2) establishes that the density variation on each edge is a consequence of a discrepancy between the outgoing flow and the incoming flow on the same edge. The former is captured by the term $f(\rho)$ whereas the latter is represented by $\tilde{B}^{T}(\alpha) \hat{B} f(\rho)$. Then density variation depends on the difference $\tilde{B}^{T}(\alpha) \hat{B} f(\rho)-f(\rho)$ which gives (2). Note that $\tilde{B}^{T}(\alpha)$ is a column (left) stochastic matrix, i.e., $\sum_{i=1, \cdots, m}\left(\tilde{B}^{T}(\alpha)\right)_{i j}=1$ for all $j=1, \cdots, n$. Also note that the relation of the state $X$ and the density $\rho$ is the classical forward Kolmogorov equation in (2) for discrete state space continuous-time Markov chains. The Kolmogorov equation represents the macroscopic dynamics. Both $\alpha$ and $\phi$ appearing in the microscopic dynamics (1) are present in the macroscopic dynamics (2). In addition, note that $\rho$ can be interpreted in two ways, as a controlled state process, and as a density under optimal control.

Assume that the graph is acyclic, and has one source node $s$ and one destination node $d$. Select a subset of paths from $s$ to $d$ and call it $\mathcal{P}$. Each element of $\mathcal{P}$ is an $s-d$ path $\{s, \cdots, i, \cdots, d\}$. Let the matrix $C \in\{0,1\}^{|\mathcal{P}| \times m}$ be given which relates paths to edges. Each row of $C$ contains ones or zeros depending on which edges are included in the path. We can define the output vector-valued function $y(\cdot): \mathbb{R}_{+} \rightarrow$ $\mathbb{R}^{|\mathcal{P}|}, t \mapsto y(t)$, which represents the collective density on each path and can be expressed as $y(t)=C \rho(t)$.

We assume that the time to cross an edge is proportional to the congestion on that edge (measured by the density). Thus the sum of densities in a path is the total time from origin to destination using that path. Based on this, in order to achieve a Wardrop equilibrium, i.e., uniform distribution over 
below, where $\mathcal{M}$ is the consensus set/Wardrop equilibrium set:

$$
\begin{array}{r}
g(x, \rho)=\operatorname{dist}(\rho, \mathcal{M}), \\
\mathcal{M}=\{\rho \in \mathcal{D}: y=C \rho=\mathbf{1} p, \text { for any } p \in[0,1]\} .
\end{array}
$$

Here $\operatorname{dist}(\rho, \mathcal{M})$ denotes the distance from the vector $\rho$ to the set $\mathcal{M}$, and 1 denotes the $|\mathcal{P}|$-dimensional column vector of ones. Actually, the value of the consensus density is the point in the consensus set which is closest to the current density vector $\rho$. Put differently, this point is the projection of $\rho$ on the consensus set $M$.

The problem in its generic form is then the following:

Problem 1: Design a routing policy to minimize the output disagreement, i.e., each player solves the following problem:

$$
\left\{\begin{array}{l}
\inf _{\alpha(\cdot)} J(x, \alpha(\cdot), \rho[\cdot](\cdot)), \\
J(\cdot)=\mathbb{E}\left[\int_{0}^{T} g(X(\tau), \rho(\tau)) d \tau+g(X(T), \rho(T))\right] \\
\{X(t), t \geq 0\} \text { as in }(1), \\
X(0)=x
\end{array}\right.
$$

where $\alpha(\cdot)$ is a measurable function taking values in $\mathcal{U}$ for any $t \in[0, T]$. A challenging aspect of the above problem is that every player minimizes a cost functional which depends on the density function of the whole population. Thus, microscopic and macroscopic representations of the system are strongly intertwined which makes the problem differ from classical optimal control or differential game theory.

Remark 1: We assume a common cost functional for all players. This cost functional depends only on the density vector. This is typical of mean-field games with global interaction. The presented model can be specialized to the case of local interaction as follows. Assume $x=e$ (pedestrian on edge $e)$, then the cost functional integrates the square deviation $\left(\rho_{e}-y_{e}\right)^{2}$ where $y$ is the projection of $\rho$ on $M$ and $y_{e}$ is its eth component, and $\rho_{e}$ is the density in the eth edge where the pedestrian lives.

Remark 2: In a practical routing model typically have different source and destination nodes. This will cause some heterogeneity to the players, and can further affect the density flow's dynamics. To account for heterogeneity we would need to reformulate our model as a multi-population game involving a Kolmogorov equation for each population. Multi-population games are a key direction for future research.

\section{MAIN RESUlTS}

In this section we highlight a few main results. First, we provide a mean-field game formulation of the problem. Second, we illustrate an extended state space solution approach. Third, we study the stochastic case where the density evolution is driven by a Brownian motion. Fourth, we investigate the case where the density evolution is perturbed by a bounded adversarial disturbance. Here we provide two equivalent convergence conditions: the first condition mirrors an already established condition in repeated games with vector payoffs, while the second condition is expressed in terms of set inclusion.

\section{A. Mean-field game formulation}

Let us denote by $v(x, t)$ the value of the optimization problem (5) starting from time $t$ at state $x$. The first step is to show that the problem results in the following mean-field game system for the unknown scalar functions $v(x, t)$ and $\rho(t)$ when each player behaves according to (5):

Lemma 1: The mean-field system for the routing problem in Problem 1 takes the form:

$$
\left\{\begin{array}{l}
\dot{v}(x, t)+\mathcal{H}(x, \Delta(v), t)=0 \text { in } E \times[0, T[ \\
v(x, T)=g(x, \rho(T)), \forall x \in E, \\
\dot{\rho}(t)=\left(\tilde{B}^{T}\left(\alpha^{*}\right) \hat{B}-I\right) f(\rho) \text { in }[0, T[ \\
\rho(0)=\rho_{0}, \rho_{0} \text { given, }
\end{array}\right.
$$

where $\mathcal{H}(x, \Delta(v), t)$ is the Hamiltonian function given by

$$
\begin{gathered}
\mathcal{H}(x, \Delta(v), t)=\inf _{\alpha \in \mathcal{U}}\left\{\sum_{z \in E} q_{x z}(v(z, t)\right. \\
-v(x, t))+g(x, \rho)\} .
\end{gathered}
$$

In the expression above, $\Delta(v)$ denotes the difference of the value function computed in two successive states and $q_{x z}$ is the transition rate given in (1). Successive states are the future edges touched by the player along his path from source to destination. The optimal time-varying control $\alpha^{*}(x, t)$ is given by

$$
\begin{aligned}
\alpha^{*}(x, t) \in & \arg \min _{\alpha \in \mathcal{U}}\left\{\sum_{z \in E} q_{x z}(v(z, t)\right. \\
& -v(x, t))+g(x, \rho)\} .
\end{aligned}
$$

Proof: Let us start by noting that the third and fourth equations of (6) are the forward Kolmogorov equation and the corresponding boundary condition on the initial distribution law. To prove the first equation of (6), we know that from dynamic programming the following holds:

$$
\begin{gathered}
\dot{v}(x, t)+\inf _{\alpha \in \mathcal{U}}\left\{\sum_{z \in E} q_{x z}(v(z, t)-v(x, t))\right. \\
+g(x, \rho)\}=0 \text { in } E \times[0, T[.
\end{gathered}
$$

By introducing the Hamiltonian $\mathcal{H}(x, \Delta(v), t)$ given in (7), we obtain the first equation. Note that the transition rates depend on the routing policy/control $\alpha$. This is then obtained as the minimizer in the computation of the Hamiltonian as expressed by (8). For the first part of the proof, note that the second equation is the boundary condition on the terminal penalty.

The mean-field game, system (6), appears in the form of two coupled ODEs intertwined in a forward-backward way. The first equation in (6) is the Hamilton-Jacobi-Bellman (HJB) equation with variable $v(x, t)$ and parametrized in $\rho(\cdot)$. Given the boundary condition on final state (second equation in (6)), and assuming a given population behavior captured by $\rho(\cdot)$, the HJB equation is solved backwards and returns the value function and best-response behavior of the players given by (8). The HJB equation is coupled with a second ODE, which is the Fokker-Planck-Kolmogorov (FPK) (third equation in (6)), defined on variable $\rho(\cdot)$ and parametrized in $\alpha^{*}(x, t)$. Given the boundary condition on initial distribution $\rho(0)=\rho_{0}$ (fourth equation in (6)), and assuming a given individual behavior described by $\alpha^{*}$, the FPK equation is solved forward and returns the population behavior time evolution $\rho(t)$. 


\section{B. State space extension}

To solve Problem 1 and the related mean-field game (6), each player needs to predict the evolution of the density over the horizon. Our solution approach to (6) involves extending the state space including $\rho$ as an additional state variable as done in [7]. This method is alternative to classical fixed-point methods. The resulting problem is of the form

$$
\begin{aligned}
\inf _{\alpha(\cdot)} & J(x, \alpha(\cdot), \rho[\cdot](\cdot)) \\
& =\mathbb{E}\left[\int_{0}^{T} g(X(\tau), \rho(\tau)) d \tau+g(X(T), \rho(T))\right],
\end{aligned}
$$

subject to $\{X(t), t \geq 0\}$ as in (1),

$$
\dot{\rho}(t)=\left(\tilde{B}^{T}(\alpha) \hat{B}-I\right) f(\rho) .
$$

Then, we look for a new value function $V(x, \rho, t)$ which depends not only on $x$ but also on the density vector $\rho$. Put differently, $v$ and $V$ live in different domains. Function $v$ lives in the state space of each single particle (the pedestrian), and is parametrized in the common state $\rho$, thus we denote it by $v(x, t)$. Function $V$ lives in the extended state space involving the state of the particle and the common state $\rho$, thus we denote it by $V(x, \rho, t)$.

With the above reasoning in mind, the mean-field system of the problem at hand can be rewritten as follows.

Lemma 2: The mean-field system for the routing problem in Problem 1 in extended form appears as:

$$
\left\{\begin{array}{l}
\partial_{t} V(x, \rho, t)+\tilde{\mathcal{H}}\left(x, \rho, \Delta(v), \partial_{\rho} V, t\right)=0 \\
\text { in } E \times[0,1]^{m} \times[0, T[, \\
V(x, \rho, T)=g(x, \rho(T)), \forall(x, \rho) \in E \times[0,1]^{m},
\end{array}\right.
$$

where

$$
\begin{aligned}
& \tilde{\mathcal{H}}\left(x, \rho, \Delta(v), \partial_{\rho} V, t\right) \\
& =\inf _{\alpha \in \mathcal{U}}\left\{\sum_{z \in E} q_{x z}(V(z, \rho, t)-V(x, \rho, t))\right. \\
& \left.+\partial_{\rho} V(x, \rho, t)^{T}\left[\left(\tilde{B}^{T}(\alpha) \hat{B}-I\right) f(\rho)\right]+g(x, \rho)\right\},
\end{aligned}
$$

and the optimal time-varying control $\alpha^{*}(x, \rho, t)$ is given by

$$
\begin{aligned}
& \alpha^{*}(x, \rho, t) \\
& \in \arg \min _{\alpha \in \mathcal{U}}\left\{\sum_{z \in E} q_{x z}(V(z, \rho, t)-V(x, \rho, t))\right. \\
& \left.+\partial_{\rho} V(x, \rho, t)\left[\left(\tilde{B}^{T}(\alpha) \hat{B}-I\right) f(\rho)\right]+g(x, \rho)\right\} .
\end{aligned}
$$

Proof: From dynamic programming we obtain

$$
\begin{gathered}
\partial_{t} V(x, \rho, t)+\inf _{\alpha \in \mathcal{U}}\left\{\sum_{z \in E} q_{x z}(V(z, \rho, t)-V(x, \rho, t))\right. \\
\left.+\partial_{\rho} V(x, \rho, t)^{T}\left[\left(\tilde{B}^{T}(\alpha) \hat{B}-I\right) f(\rho)\right]+g(x, \rho)\right\}=0 \\
\text { in } E \times[0,1]^{m} \times[0, T[.
\end{gathered}
$$

By introducing the Hamiltonian $\tilde{\mathcal{H}}\left(x, \rho, \Delta(v), \partial_{\rho} V, t\right)$ given in (10), the first equation is proven. To prove (11), observe that the optimal control is the minimizer in the computation of the extended Hamiltonian. It remains to note that the second equation in (9) is the boundary condition on the terminal penalty.

Assumption 1: Let $\mathcal{M}$ be given by (4), $r>0$ and $U=$ $\left\{\rho \in \mathbb{R}^{m}: \operatorname{dist}(\rho, \mathcal{M})<r\right\}$. For all $\rho \in U \backslash \mathcal{M}$ there exists $y \in \Pi_{\mathcal{M}}(\rho)$, where $\Pi_{\mathcal{M}}(\rho)$ is the projection of $\rho$ on the set
$\mathcal{M}$, such that the value $\operatorname{val}[\lambda]$ is negative for every $\lambda=\rho-y$, i.e.,

$$
\begin{aligned}
& \operatorname{val}[\lambda]=\inf _{\alpha}\left\{\lambda^{T} \dot{\rho}\right\} \\
& \quad=\inf _{\alpha \in \mathcal{U}}\left\{\sum_{z \in E} \dot{\rho}_{z} \lambda_{z}\right\}<0, \forall \lambda=\rho-y .
\end{aligned}
$$

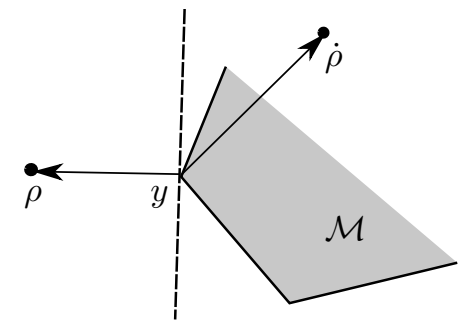

Fig. 2: Geometric illustration of the attainability condition.

This assumption ensures that for a given feasible target set, there always exists a routing policy $\alpha(t)$ that drives the edge density $\rho$ towards the set ( $\lambda$ can be viewed as the vector connecting the current density projection point on the target set and the current density point, with the direction pointing out from the target set). This is illustrated in Fig. 2. We can then establish the following result.

Lemma 3: Let Assumption 1 hold true. Then the mean-field game for the routing problem in Problem 1 is given by

$$
\left\{\begin{array}{l}
\partial_{t} V(x, \rho, t)+\operatorname{val}\left[\partial_{\rho} V(x, \rho, t)\right]+g(x, \rho)=0 \\
\text { in } E \times[0,1]^{m} \times[0, T[, \\
V(x, \rho, T)=g(x, \rho(T)), \forall(x, \rho) \in E \times[0,1]^{m} .
\end{array}\right.
$$

Furthermore, the optimal control is:

$$
\begin{array}{r}
\alpha^{*}(x, \rho, t)=\arg \min _{\alpha \in \mathcal{U}}\left\{\partial_{\rho} V(x, \rho, t)^{T}\right. \\
\left.\cdot\left[\left(\tilde{B}^{T}(\alpha) \hat{B}-I\right) f(\rho)\right]\right\} .
\end{array}
$$

Proof: From (12) we have

$$
\begin{aligned}
& \operatorname{val}\left[\partial_{\rho} V(x, \rho, t)\right]=\inf _{\alpha \in \mathcal{U}}\left\{\partial_{\rho} V(x, \rho, t)^{T} \dot{\rho}\right\} \\
& =\inf _{\alpha \in \mathcal{U}}\left\{\partial_{\rho} V(x, \rho, t)^{T}\left[\left(\tilde{B}^{T}(\alpha) \hat{B}-I\right) f(\rho)\right]\right\} \\
& =\tilde{\mathcal{H}}\left(x, \rho, \Delta(v), \partial_{\rho} V, t\right)-g(x, \rho) .
\end{aligned}
$$

Invoking Lemma 2, and the first equation in (9), we obtain the first equation in (13). The second equation in (13) is again the boundary condition on the terminal penalty. It remains to note that the optimal control is the minimizer in the computation of the extended Hamiltonian and thus is obtained from (14).

A slight modification of Assumption 1 which leads to exponential convergence is as follows.

Assumption 2: Let $\mathcal{M}$ be given as in (4), $r>0$ and $U=$ $\left\{\rho \in \mathbb{R}^{m}: \operatorname{dist}(\rho, \mathcal{M})<r\right\}$. For all $\rho \in U \backslash \mathcal{M}$ there exists $y \in \Pi_{\mathcal{M}}(\rho)$ such that the value $\operatorname{val}[\lambda]$, is negative for every $\lambda=\rho-y$, i.e.,

$$
\begin{aligned}
& \operatorname{val}[\lambda]=\inf _{\alpha \in \mathcal{U}}\left\{\lambda^{T} \dot{\rho}\right\} \\
& =\inf _{\alpha \in \mathcal{U}}\left\{\sum_{z \in E} \dot{\rho}_{z} \lambda_{z}\right\}<-\kappa \lambda^{T} \lambda, \forall \lambda=\rho-y
\end{aligned}
$$

for a positive scalar $\kappa$. 
The condition in Assumption 1 guarantees that a preassigned set $\mathcal{M}$ is attainable. However, any set can be attainable under a stronger condition which we copy and readapt from [6], [26].

Assumption 3: The value $\operatorname{val}[\lambda]$, is negative for every $\lambda \in$ $\mathbb{R}^{m}$, i.e.,

$$
\begin{aligned}
& \operatorname{val}[\lambda]=\inf _{\alpha \in \mathcal{U}}\left\{\lambda^{T} \dot{\rho}\right\} \\
& =\inf _{\alpha \in \mathcal{U}}\left\{\sum_{z \in E} \dot{\rho}_{z} \lambda_{z}\right\}<0, \forall \lambda \in \mathbb{R}^{m} .
\end{aligned}
$$

In the following, Assumptions 1-3 are reviewed both in a stochastic and a worst-case framework. Such conditions are proven to be sufficient conditions for different types of convergence (exponential, almost surely).

\section{Stochastic case}

In this section, we analyze the case where the density evolves according to a stochastic differential equation driven by a Brownian motion. The Kolmogorov equation is then replaced by a geometric Brownian motion dynamics as illustrated below:

$$
d \rho(t)=\left(\tilde{B}^{T}(\alpha) \hat{B}-I\right) f(\rho) d t+\sigma \operatorname{dist}(\rho, \mathcal{M}) d \mathcal{B}(t) .
$$

Note that now $\rho$ is in $\mathbb{R}^{m}$ and not in $\mathcal{D}$ as the conservation of mass is no longer preserved. This means that we can have births or deaths in the population. Extending the state space as in the earlier case, and introducing the extended Hamiltonian for the stochastic case as

$$
\begin{aligned}
& \tilde{\mathcal{H}}\left(x, \rho, \Delta(v), \partial_{\rho} V, t\right) \\
& =\inf _{\alpha \in \mathcal{U}}\left\{\sum_{z \in E} q_{x z}(V(z, \rho, t)-V(x, \rho, t))\right. \\
& \left.+\partial_{\rho} V(x, \rho, t)^{T}\left[\left(\tilde{B}^{T}(\alpha) \hat{B}-I\right) f(\rho)\right]+g(x, \rho)\right\},
\end{aligned}
$$

the mean-field system turns into the system of equations below in the value function $V(x, \rho, t)$ in $E \times \mathbb{R}^{m} \times[0, T[$ :

$$
\left\{\begin{array}{l}
\partial_{t} V(x, \rho, t)+\tilde{\mathcal{H}}\left(x, \rho, \Delta(v), \partial_{\rho} V, t\right) \\
+\frac{\sigma^{2}}{2} \operatorname{dist}^{2}(\rho, \mathcal{M}) \operatorname{Tr}\left(\partial_{\rho \rho}^{2} V(x, \rho, t)\right)=0 \\
\text { in } E \times \mathbb{R}^{m} \times[0, T[ \\
V(x, \rho, T)=g(x, \rho(T)), \forall(x, \rho) \in E \times \mathbb{R}^{m}
\end{array}\right.
$$

where the optimal time-varying control $\alpha^{*}(x, \rho, t)$ is obtained as

$$
\begin{gathered}
\alpha^{*}(x, \rho, t) \in \arg \min _{\alpha \in \mathcal{U}}\left\{\sum_{z \in E} q_{x z}(V(z, \rho, t)-V(x, \rho, t))+\right. \\
\left.\partial_{\rho} V(x, \rho, t)^{T}\left[\left(\tilde{B}^{T}(\alpha) \hat{B}-I\right) f(\rho)\right]+g(x, \rho)\right\} .
\end{gathered}
$$

Assumption 4: (Attainability condition) Let $\mathcal{M}$ be given as in (4), $r>0$ and $U=\left\{\rho \in \mathbb{R}^{m}: \operatorname{dist}(\rho, \mathcal{M})<r\right\}$. For all $\rho \in U \backslash \mathcal{M}$ there exists $y \in \Pi_{\mathcal{M}}(\rho)$ such that the value expval $[\lambda]$ is negative for every $\lambda=\rho-y$, i.e.,

$$
\begin{aligned}
& \text { expval }[\lambda] \\
& =\inf _{\alpha \in \mathcal{U}}\left\{\lambda^{T}\left[\left(\tilde{B}^{T}(\alpha) \hat{B}-I\right) f(\rho)\right]\right\} \\
& =\inf _{\alpha \in \mathcal{U}}\left\{\sum_{z \in E}\left[\left(\tilde{B}^{T}(\alpha) \hat{B}-I\right) f(\rho)\right]_{z} \lambda_{z}\right\} \\
& <-\sigma^{2} m \operatorname{dist}^{2}(\rho, \mathcal{M}), \forall \lambda=\rho-y,
\end{aligned}
$$

where $\left[\left(\tilde{B}^{T}(\alpha) \hat{B}-I\right) f(\rho)\right]_{z}$ denotes the $z$ th component of vector $\left(\tilde{B}^{T}(\alpha) \hat{B}-I\right) f(\rho)$.

We can then establish the following result.

Lemma 4: Let Assumpion 4 hold true. Then, the mean-field game for the routing problem in Problem 1 in the stochastic case is given by

$$
\left\{\begin{array}{l}
\partial_{t} V(x, \rho, t)+\text { expval }\left[\partial_{\rho} V(x, \rho, t)\right]+g(x, \rho) \\
+\frac{\sigma^{2}}{2} \operatorname{dist}^{2}(\rho, \mathcal{M}) \operatorname{Tr}\left(\partial_{\rho \rho}^{2} V(x, \rho, t)\right)=0 \\
\text { in } E \times \mathbb{R}^{m} \times[0, T[ \\
V(x, \rho, T)=g(x, \rho(T)), \forall(x, \rho) \in E \times \mathbb{R}^{m}
\end{array}\right.
$$

Furthermore, the optimal control is:

$$
\begin{aligned}
\alpha^{*}(x, \rho, t)= & \arg \min _{\alpha \in \mathcal{U}}\left\{\partial_{\rho} V(x, \rho, t)^{T}\right. \\
& \left.\cdot\left[\left(\tilde{B}^{T}(\alpha) \hat{B}-I\right) f(\rho)\right]\right\} .
\end{aligned}
$$

Proof: Let us observe that from (20) we have

$$
\begin{aligned}
& \text { expval }\left[\partial_{\rho} V(x, \rho, t)\right]=\inf _{\alpha \in \mathcal{U}}\left\{\partial_{\rho} V(x, \rho, t)^{T} d \rho\right\} \\
& =\inf _{\alpha \in \mathcal{U}}\left\{\partial_{\rho} V(x, \rho, t)^{T}\left[\left(\tilde{B}^{T}(\alpha) \hat{B}-I\right) f(\rho)\right]\right\} \\
& =\tilde{\mathcal{H}}\left(x, \rho, \Delta(v), \partial_{\rho} V, t\right)-g(x, \rho) .
\end{aligned}
$$

From the above equation and invoking the first equation in (19), we obtain the first equation in (21). The second equation in (21) represents the boundary condition on the terminal penalty. To conclude our proof we notice that the optimal control is the minimizer in the computation of the extended Hamiltonian and thus is obtained from (22).

Let us now take $T \rightarrow \infty$, and consider the corresponding infinite horizon problem. The value function is now rewritten as $V(\rho(t))$ as it is no longer explicitly dependent on time (dependence on time is only through the vector $\rho(t)$ ). In addition to this, in the proof of the next result, the value function is reviewed as a Lyapunov function. We are in the position to establish the next result, regarding the stability almost surely of the stochastic process at hand.

Theorem 1: (Stability almost surely) Let Assumption 4 hold true. Then, dynamics (17) converges exponentially to $\mathcal{M}$ almost surely, namely

$$
\operatorname{dist}(\rho(t), \mathcal{M})=e^{-t} \operatorname{dist}(\rho(0), \mathcal{M}), \quad \text { with probability } 1 .
$$

Proof: Let $\rho$ be a solution of dynamics (17) with initial value $\rho(0) \in U \backslash \mathcal{M}$. Set $\tau=\{\inf t>0 \mid \rho(t) \in \mathcal{M}\} \leq \infty$ and let $V(\rho(t))=\operatorname{dist}(\rho(t), \mathcal{M})$. For all $t \in[0, \tau]$ and $y \in$ $\Pi_{\mathcal{M}}(\rho(t))$

$$
\begin{aligned}
& V(\rho(t+d t))-V(\rho(t))=\|\rho(t+d t)-y\|-\|\rho(t)-y\| \\
& =\|\rho(t)+d \rho(t)-y\|-\|\rho(t)-y\| \\
& =\frac{1}{\|\rho(t)+d \rho(t)-y\|}\|\rho(t)+d \rho(t)-y\|^{2}-\frac{1}{\|\rho(t)-y\|}\|\rho(t)-y\|^{2} .
\end{aligned}
$$


From the definition of infinitesimal generator

$$
\begin{aligned}
& \mathcal{L} V(\rho(t))=\lim _{d t \rightarrow 0} \frac{V(\rho(t+d t))-V(\rho(t))}{d t} \\
& =\lim _{d t \rightarrow 0} \frac{1}{d t}\left[\frac{1}{\|\rho(t)+d \rho(t)-y\|}\|\rho(t)+d \rho(t)-y\|^{2}\right. \\
& \left.\quad-\frac{1}{\|\rho(t)-y\|}\|\rho(t)-y\|^{2}\right] \\
& =\lim _{d t \rightarrow 0} \frac{1}{d t}\left[\frac{1}{\|\rho(t)-y\|+O(\sqrt{d t})}\|\rho(t)+d \rho(t)-y\|^{2}\right. \\
& \left.\quad-\frac{1}{\|\rho(t)-y\|}\|\rho(t)-y\|^{2}\right] \\
& =\frac{1}{\|\rho(t)-y\|} \lim _{d t \rightarrow 0} \frac{1}{d t}\left[\|\rho(t)+d \rho(t)-y\|^{2}\right. \\
& =\frac{1}{\|\rho(t)-y\|} \mathcal{L}\|\rho(t)-y\|^{2} \\
& \leq \frac{2}{\|\rho(t)-y\|}\left[\inf _{\alpha}\left\{(\rho(t)-y)^{T}\left(\tilde{B}^{T}(\alpha) \hat{B}-I\right) f(\rho)\right\}\right. \\
& \left.\quad+\frac{\sigma^{2}}{2} d i s t^{2}(\rho, \mathcal{M}) \operatorname{Tr}\left(\partial_{\rho \rho}^{2}\|\rho(t)-y\|^{2}\right)\right] \\
& \leq \frac{2}{\|\rho(t)-y\|}\left[\operatorname{expval}^{2}[\rho(t)-y]+\sigma^{2} m \operatorname{dist}(\rho, \mathcal{M})\right] .
\end{aligned}
$$

Now, from Assumption 4 we have that for all $\rho \in U \backslash \mathcal{M}$ there exists $y \in \Pi_{\mathcal{M}}(\rho)$ such that

$$
\text { expval }[\rho(t)-y]+\sigma^{2} m \operatorname{dist}^{2}(\rho, \mathcal{M})<0,
$$

which implies $\mathcal{L} V(\rho(t))<0$, for all $\rho(t) \in U \backslash \mathcal{M}$ and this concludes our proof.

Similar convergence results can be established in the case where the density evolution is subject to a bounded adversarial disturbance.

\section{Adversarial disturbance}

In the presence of an adversarial disturbance the density evolution expressed by dynamics (2) takes the form

$$
\dot{\rho}(t)=\left(\tilde{B}^{T}(\alpha, \omega) \hat{B}-I\right) f(\rho)
$$

where $\omega$ is the disturbance. We assume that the disturbance $\omega$ is bounded and belongs to polytope

$$
\begin{gathered}
\mathcal{W}=\left\{\omega \in[-1,1]^{m} \mid\left\{\omega_{j}\right\}_{j \in \varepsilon^{+}(i)} \in \Delta_{0}^{\left|\varepsilon^{+}(i)\right|},\right. \\
\text { for all } i=1, \ldots, n,\}
\end{gathered}
$$

where $\Delta_{0}^{\left|\varepsilon^{+}(i)\right|}$ is the simplex translated to the origin in $\mathbb{R}^{\left|\varepsilon^{+}(i)\right|}$. In other words, the above corresponds to the constraint $\sum_{j \in \varepsilon^{+}(i)} \omega_{j}=0$ for all $i=1, \ldots, n$.

The robust version of Problem 1, involving the adversarial disturbance as described is then the following:

Problem 2: Design a routing policy to minimize the output disagreement, i.e., each player solves the following problem:

$$
\left\{\begin{array}{l}
\inf _{\alpha(\cdot)} \sup _{\omega(\cdot)} J(x, \alpha(\cdot), \rho[\cdot](\cdot)), \\
J(\cdot)=\mathbb{E}\left[\int_{0}^{T} g(X(\tau), \rho(\tau)) d \tau+g(X(T), \rho(T))\right], \\
\{X(t), t \geq 0\} \text { as in }(1), \\
\dot{\rho}(t)=\left(\tilde{B}^{T}(\alpha, \omega) \hat{B}-I\right) f(\rho),
\end{array}\right.
$$

where $\alpha(\cdot)$ and $\omega(\cdot)$ are measurable functions taking values in $\mathcal{U}$ and $\mathcal{W}$. In the above problem, every player minimizes a cost functional which depends on the density function of the whole population. The density in turn depends on both the control $\alpha(\cdot)$ and the disturbance $\omega(\cdot)$. Let us denote by $v(x, t)$ the value of the optimization problem starting from time $t$ at state $x$. Similarly to the case of Lemma 1, we can derive the following mean-field system:

Theorem 2: The mean-field system for the routing problem in Problem 2 takes on the form:

$$
\left\{\begin{array}{l}
\dot{v}(x, t)+\mathcal{H}(x, \Delta(v), t)=0 \text { in } E \times[0, T[ \\
v(x, T)=g(x, \rho(T)), \forall x \in E \\
\dot{\rho}(t)=\left(\tilde{B}^{T}\left(\alpha^{*}, \omega^{*}\right) \hat{B}-I\right) f(\rho) \text { in }[0, T[ \\
\rho(0)=\rho_{0}, \rho_{0} \text { given, }
\end{array}\right.
$$

$\mathcal{H}(x, \Delta(v), t)$ is the robust Hamiltonian function given by

$$
\begin{aligned}
& \mathcal{H}(x, \Delta(v), t)= \\
& \inf _{\alpha \in \mathcal{U}} \sup _{\omega \in \mathcal{W}}\left\{\sum_{z \in E} q_{x z}(v(z, t)-v(x, t))+g(x, \rho)\right\} .
\end{aligned}
$$

In the expression above, $\Delta(v)$ denotes the difference of the value function computed in two successive states, $q_{x z}$ is the transition rate given in (1). The optimal time-varying control $\alpha^{*}(x, t)$ is given by

$$
\begin{aligned}
& \alpha^{*}(x, t) \in \arg \min _{\alpha \in \mathcal{U}}\left\{\sum_{z \in E} q_{x z}(v(z, t)-v(x, t))\right. \\
& +g(x, \rho)\}, \\
& \begin{aligned}
\omega^{*}(x, t) \in \arg \max _{\omega \in \mathcal{W}}\left\{\sum_{z \in E} q_{x z}(v(z, t)-v(x, t))\right. \\
+g(x, \rho)\} .
\end{aligned}
\end{aligned}
$$

Proof: Let us start by noting that the third and fourth equations of (25) are the forward Kolmogorov equation and the corresponding boundary condition on the initial distribution law. To prove the first equation of (25), we know that from dynamic programming it holds:

$$
\begin{gathered}
\dot{v}(x, t)+\inf _{\alpha \in \mathcal{U}} \sup _{\omega \in \mathcal{W}}\left\{\sum_{z \in E} q_{x z}(v(z, t)-v(x, t))\right. \\
+g(x, \rho)\}=0 \text { in } E \times[0, T[
\end{gathered}
$$

By introducing the robust Hamiltonian $\mathcal{H}(x, \Delta(v), t)$ given in (26), we obtain the first equation. Note that the transition rates depend on the routing policy/control $\alpha$. This is then obtained as the minimizer in the computation of the Hamiltonian as expressed by (27). For the first part of the proof, please note that the second equation is the boundary condition on the terminal penalty.

Extending the state space and introducing the robust Hamiltonian in the extended space we obtain

$$
\begin{aligned}
& \tilde{\mathcal{H}}\left(x, \rho, \Delta(v), \partial_{\rho} V, t\right) \\
& =\inf _{\alpha \in U} \sup _{\omega \in \mathcal{W}}\left\{\sum_{z \in E} q_{x z}(V(z, \rho, t)-V(x, \rho, t))\right. \\
& \left.+\partial_{\rho} V(x, \rho, t)^{T}\left[\left(\tilde{B}^{T}(\alpha, \omega) \hat{B}-I\right) f(\rho)\right]+g(x, \rho)\right\} .
\end{aligned}
$$

Then the mean-field system turns into the system of equations below in the value function $V(x, \rho, t)$ in $E \times[0,1]^{m} \times[0, T[$,

$$
\left\{\begin{array}{l}
\partial_{t} V(x, \rho, t)+\tilde{\mathcal{H}}\left(x, \rho, \Delta(v), \partial_{\rho} V, t\right)=0 \\
\text { in } E \times[0,1]^{m} \times[0, T[, \\
V(x, \rho, T)=g(x, \rho(T)) \forall(x, \rho) \in E \times[0,1]^{m},
\end{array}\right.
$$


where the optimal time-varying state-feedback controls $\alpha^{*}(x, t)$ is obtained as

$$
\begin{array}{r}
\alpha^{*}(x, t) \in \arg \min _{\alpha \in \mathcal{U}}\left\{\sum_{z \in E} q_{x z}(V(z, \rho, t)-V(x, \rho, t))\right. \\
\left.+\partial_{\rho} V(x, \rho, t)^{T}\left[\left(\tilde{B}^{T}\left(\alpha, \omega^{*}\right) \hat{B}-I\right) f(\rho)\right]+g(x, \rho)\right\}
\end{array}
$$

and the worst-case adversarial disturbance is given by

$$
\begin{aligned}
& \omega^{*}(x, t) \in \arg \sup _{\omega \in \mathcal{W}}\left\{\sum_{z \in E} q_{x z}(V(z, \rho, t)-V(x, \rho, t))\right. \\
& \left.+\partial_{\rho} V(x, \rho, t)^{T}\left[\left(\tilde{B}^{T}\left(\alpha^{*}, \omega\right) \hat{B}-I\right) f(\rho)\right]+g(x, \rho)\right\} .
\end{aligned}
$$

Let us denote by $\operatorname{Ext}\{\mathcal{U}\}$ and $\operatorname{Ext}\{\mathcal{W}\}$ the set of the indices of all the vertices of $\mathcal{U}$ and $\mathcal{W}$ respectively. Let us denote the generic vertex of $\mathcal{U}$ and $\mathcal{W}$ by $a^{(k)}$ and $w^{(k)}$, respectively. Also, let us assume that $|\operatorname{Ext}\{\mathcal{U}\}|=p$ and $|\operatorname{Ext}\{\mathcal{W}\}|=q$, where $|\operatorname{Ext}\{\mathcal{U}\}|$ and $|\operatorname{Ext}\{\mathcal{W}\}|$ are the cardinality of $\operatorname{Ext}\{\mathcal{U}\}$ and $\operatorname{Ext}\{\mathcal{W}\}$, respectively. Then $\mathcal{U}=\operatorname{hull}\left\{a^{(k)}, k \in \operatorname{Ext}\{\mathcal{U}\}\right\}$, and $\mathcal{W}=\operatorname{hull}\left\{w^{(k)}, k \in\right.$ $\operatorname{Ext}\{\mathcal{W}\}\}$ where hull stands for the convex hull.

From the Caratheodory theorem we know that any point in the polytope can be expressed as convex combination of a subset of vertices, namely

$$
\begin{aligned}
\alpha=\sum_{k \in \operatorname{Ext}\{\mathcal{U}\}} a_{k} a^{(k)}, \sum_{k \in \operatorname{Ext}\{\mathcal{U}\}} a_{k}=1 \\
\omega=\sum_{k \in \operatorname{Ext}\{\mathcal{W}\}} w_{k} w^{(k)}, \quad \sum_{k \in \operatorname{Ext}\{\mathcal{W}\}} w_{k}=1 .
\end{aligned}
$$

In other words, $a_{k}$ is the weight of the convex combination $\sum_{k \in E x t\{\mathcal{U}\}} a_{k} a^{(k)}$ and similarly $w_{k}$ is the weight of the convex combination $\sum_{k \in \operatorname{Ext}\{\mathcal{W}\}} w_{k} w^{(k)}$.

This is useful, as we can now review $\alpha$ and $\omega$ as mixed strategies of the two-player game with vector payoffs displayed in Table I.

The density at time $t$ is now obtained as the cumulative payoff up to time $t$, namely

$$
\left\{\begin{array}{l}
\rho(t)=\int_{0}^{t} \dot{\rho}(\tau) d \tau=\int_{0}^{t}\left(\tilde{B}^{T}(\alpha, \omega) \hat{B}-I\right) f(\rho) d \tau, \\
\rho(0)=\rho_{0} .
\end{array}\right.
$$

Convergence conditions on the cumulative payoffs are connected to the value of the projected game. The projected game, which is displayed in Table II, is obtained by premultiplying each entry by a given vector $\lambda \in \mathbb{R}^{m}$.

The above is a classical two-player game with scalar payoffs and therefore it admits a value given by

$$
\operatorname{val}[\lambda]:=\inf _{\alpha} \sup _{\omega}\left\{\lambda^{T}\left(\tilde{B}^{T}(\alpha, \omega) \hat{B}-I\right) f(\rho)\right\} .
$$

Assumption 5: (Attainability of set $\mathcal{M}$ ) Let $\mathcal{M}$ be given as in (4), $r>0$ and $U=\left\{\rho \in \mathbb{R}^{m}: \operatorname{dist}(\rho, \mathcal{M})<r\right\}$. For all $\rho \in U \backslash \mathcal{M}$ there exists $y \in \Pi_{\mathcal{M}}(\rho)$ such that the value of the projected game, $\operatorname{val}[\lambda]$, is negative for every $\lambda=\rho-y$, i.e.,

$$
\begin{aligned}
\operatorname{val}[\lambda] & :=\inf _{\alpha} \sup _{\omega}\left\{(\rho-y)^{T}\right. \\
& \left.\cdot\left(\tilde{B}^{T}(\alpha, \omega) \hat{B}-I\right) f(\rho)\right\}<0, \forall \lambda=\rho-y .
\end{aligned}
$$

Condition (30) in Assumption 5 is as in [6], [26]. Figure 3 provides a geometric description of it.

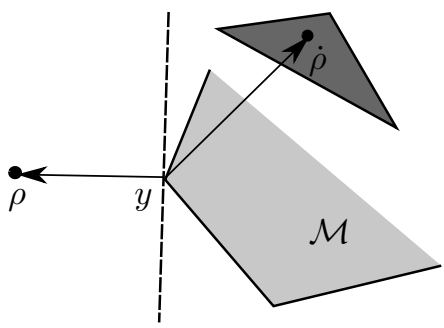

Fig. 3: Geometric illustration of the attainability condition.

This assumption ensures that for a given feasible target set, there always exists a routing policy $\alpha(t)$ that drives the edge density $\rho$ towards the set whatever the disturbance does. Again, $\lambda$ can be taken as the vector connecting the current density projection point on the target set and the current density point, with the direction pointing out from the target set. Note that checking this condition requires solving a sequence of linear or quadratic programs (find projection, construct hyperplane, and check inequality). We can then establish the following result.

Theorem 3: Let Assumption 5 hold true. Then the meanfield game for the routing problem in Problem 2 is given by

$$
\left\{\begin{array}{l}
\partial_{t} V(x, \rho, t)+\operatorname{val}\left[\partial_{\rho} V(x, \rho, t)\right]+g(x, \rho)=0 \\
\text { in } E \times[0,1]^{m} \times[0, T[, \\
V(x, \rho, T)=g(x, \rho(T)), \forall(x, \rho) \in E \times[0,1]^{m} .
\end{array}\right.
$$

Furthermore, the optimal control and worst-case disturbance are:

$$
\begin{array}{r}
\alpha^{*}(x, \rho, t)=\arg \min _{\alpha}\left\{\partial_{\rho} V(x, \rho, t)^{T}\right. \\
\left.\cdot\left[\left(\tilde{B}^{T}\left(\alpha, \omega^{*}\right) \hat{B}-I\right) f(\rho)\right]\right\}, \\
\omega^{*}(x, \rho, t)=\arg \min _{\alpha}\left\{\partial_{\rho} V(x, \rho, t)^{T}\right. \\
\left.\cdot\left[\left(\tilde{B}^{T}\left(\alpha^{*}, \omega\right) \hat{B}-I\right) f(\rho)\right]\right\} .
\end{array}
$$

Proof: From (30) we have

$$
\begin{aligned}
& \operatorname{val}\left[\partial_{\rho} V(x, \rho, t)\right]=\inf _{\alpha \in \mathcal{U}} \sup _{\omega \in \mathcal{W}}\left\{\partial_{\rho} V(x, \rho, t)^{T} \dot{\rho}\right\} \\
& =\inf _{\alpha \in \mathcal{U}} \sup _{\omega \in \mathcal{W}}\left\{\partial_{\rho} V(x, \rho, t)^{T}\left[\left(\tilde{B}^{T}(\alpha, \omega) \hat{B}-I\right) f(\rho)\right]\right\} \\
& =\tilde{\mathcal{H}}\left(x, \rho, \Delta(v), \partial_{\rho} V, t\right)-g(x, \rho) .
\end{aligned}
$$

\begin{tabular}{l||c|c|c}
$u^{(i)} / w^{(j)}$ & $w^{(1)}$ & $\ldots$ & $w^{(q)}$ \\
\hline \hline$a^{(1)}$ & $\left(\tilde{B}^{T}\left(a^{(1)}, w^{(1)}\right) \hat{B}-I\right) f(\rho)$ & $\ldots$ & $\left(\tilde{B}^{T}\left(a^{(1)}, w^{(q)}\right) \hat{B}-I\right) f(\rho)$ \\
\hline$\vdots$ & $\vdots$ & & $\vdots$ \\
\hline$a^{(p)}$ & & &
\end{tabular}

TABLE I: Two-player game with vector payoffs. 


\begin{tabular}{l||c|c|c}
$u^{(i)} / w^{(j)}$ & $w^{(1)}$ & $\ldots$ & $w^{(q)}$ \\
\hline \hline$a^{(1)}$ & $\lambda^{T}\left(\tilde{B}^{T}\left(a^{(1)}, w^{(1)}\right) \hat{B}-I\right) f(\rho)$ & $\ldots$ & $\lambda^{T}\left(\tilde{B}^{T}\left(a^{(1)}, w^{(q)}\right) \hat{B}-I\right) f(\rho)$ \\
\hline$\vdots$ & $\vdots$ & & $\vdots$ \\
\hline$a^{(p)}$ & $\vdots$ & &
\end{tabular}

TABLE II: Two-player projected game.

Invoking (28), we obtain the first equation in (31). The second equation in (31) is again the boundary condition on the terminal penalty. It remains to notice that the optimal control is the minimizer in the computation of the extended Hamiltonian and thus is obtained from (32). Analogously, the worst-case disturbance is the maximizer in the computation of the extended Hamiltonian and thus is obtained from the second equation in (32).

As for the stochastic case in the previous section, we now take $T \rightarrow \infty$, and consider the relevant infinite horizon problem. In the proof of the next theorem, the value function is now interpreted as a Lyapunov function, and as it is stationary, it is rewritten as $V(\rho(t))$.

Theorem 4: Let Assumption 5 hold true. Then, dynamics (23) converges asymptotically to $\mathcal{M}$, namely

$$
\lim _{t \rightarrow \infty} \operatorname{dist}(\rho(t), \mathcal{M})=0 .
$$

Proof: Let $\rho$ be a solution of dynamics (23) with initial value $\rho(0) \in U \backslash \mathcal{M}$. Set $\tau=\{\inf t>0 \mid \rho(t) \in \mathcal{M}\} \leq \infty$ and let $V(\rho(t))=\operatorname{dist}(\rho(t), \mathcal{M})$. For all $t \in[0, \tau]$ and $y \in$ $\Pi_{\mathcal{M}}(\rho(t))$

$$
\begin{aligned}
& V(\rho(t+d t))-V(\rho(t))=\|\rho(t+d t)-y\|-\|\rho(t)-y\| \\
& =\|\rho(t)+\dot{\rho}(t) d t-y\|-\|\rho(t)-y\|+|d t| \epsilon(d t) \\
& =\frac{1}{\|\rho(t)+\dot{\rho}(t) d t-y\|}\|\rho(t)+\dot{\rho}(t) d t-y\|^{2} \\
& \quad-\frac{1}{\|\rho(t)-y\|}\|\rho(t)-y\|^{2}+|d t| \epsilon(d t)
\end{aligned}
$$

where $\lim _{d t \rightarrow 0} \epsilon(d t)=0$. Hence

$$
\begin{aligned}
& \dot{V}(\rho(t))=\lim _{d t \rightarrow 0} \frac{1}{d t}\left(\frac{1}{\|\rho(t)+\dot{\rho}(t) d t-y\|}\|\rho(t)+\dot{\rho}(t) d t-y\|^{2}\right. \\
& \left.-\frac{1}{\|\rho(t)-y\|}\|\rho(t)-y\|^{2}+|d t| \epsilon(d t)\right) \\
& =\lim _{d t \rightarrow 0} \frac{1}{d t}\left(\frac{1}{\|\rho(t)-y\|+O(\sqrt{d t})}\|\rho(t)+\dot{\rho}(t) d t-y\|^{2}\right. \\
& \left.-\frac{1}{\|\rho(t)-y\|}\|\rho(t)-y\|^{2}+|d t| \epsilon(d t)\right) \\
& =\frac{1}{\|\rho(t)-y\|} \lim _{d t \rightarrow 0} \frac{1}{d t}\left(\|\rho(t)+\dot{\rho}(t) d t-y\|^{2}-\|\rho(t)-y\|^{2}\right) \\
& =\frac{1}{\|\rho(t)-y\|} \frac{d}{d t}\left(\|\rho(t)-y\|^{2}\right) \\
& \leq \frac{2}{\|\rho(t)-y\|}(\rho(t)-y)^{T} \dot{\rho}(t) \text {. }
\end{aligned}
$$

Now, as $\mathcal{M}$ is a compact set, from Assumption 5 we have that for all $\rho \in U \backslash \mathcal{M}$ there exists $y \in \Pi_{\mathcal{M}}(\rho)$ such that the affine hyperplane orthogonal to $[\rho(t), y]$ at $y$ separates $\rho(t)-y$ from $\dot{\rho}(t)$, namely

$$
\begin{aligned}
\operatorname{val}[\rho(t)-y]:= & \inf _{\alpha} \sup _{\omega}\left\{(\rho-y)^{T}\right. \\
& \left.\left(\tilde{B}^{T}(\alpha, \omega) \hat{B}-I\right) f(\rho)\right\} \\
= & \inf _{\alpha} \sup _{\omega}\left\{(\rho-y)^{T} \dot{\rho}(t)\right\}<0
\end{aligned}
$$

from which we have

$$
\dot{V}(\rho(t)) \leq \frac{2}{\|\rho(t)-y\|}(\rho(t)-y)^{T} \dot{\rho}(t)<0,
$$

and this concludes our proof.

The condition in Assumption 5 guarantees that a specific set $\mathcal{M}$ is attainable. However, any set can be attainable under a stronger condition which we copy and readapt from [6], [26].

Assumption 6: (General attainability) The value of the projected game, $\operatorname{val}[\lambda]$, is negative for every $\lambda \in \mathbb{R}^{m}$, i.e.,

$$
\begin{aligned}
\operatorname{val}[\lambda]:= & \inf _{\alpha} \sup _{\omega}\left\{\lambda^{T}\right. \\
& \left.\cdot\left(\tilde{B}^{T}(\alpha, \omega) \hat{B}-I\right) f(\rho)\right\}<0, \forall \lambda \in \mathbb{R}^{m} .
\end{aligned}
$$

It is worth noting that, denoting the set of all possible payoffs for a fixed mixed action $\alpha$ of player 1 (and fixed $\rho)$ by $D_{1}(\alpha)=\left\{\left(\tilde{B}^{T}(\alpha, \omega) \hat{B}-I\right) f(\rho): \omega \in \mathcal{W}\right\}$, then the attainability condition (34) implies that for every $\lambda \in \mathbb{R}^{m}$ there always exists a $\alpha$ such that $D_{1}(\alpha)$ is contained in the open half space $H:=\left\{x \in \mathbb{R}^{m} \mid \lambda^{T} x<0\right\}$ in $\mathbb{R}^{m}$ [26].

The next result provides an equivalent convergence condition in terms of set inclusion. In preparation to this, let us isolate the contribution of the control and disturbance by superposition and rewrite

$$
\tilde{B}^{T}(\alpha, \omega)=A(\alpha)+Q(\omega) .
$$

Substituting in (23) we have

$$
\dot{\rho}(t)=[(A(\alpha)+Q(\omega)) \hat{B}-I] f(\rho) .
$$

Let us denote by

$$
\begin{aligned}
& \mathcal{A}=\{A(\alpha) \mid \alpha \in \mathcal{U}\} \\
& \mathcal{Q}=\{Q(\omega) \mid \omega \in \mathcal{W}\}
\end{aligned}
$$

and consider the following set inclusion condition,

$$
\mathcal{A} \hat{B} f(\rho) \supset-\mathcal{Q B} f(\rho)+f(\rho) .
$$

Theorem 5: The set inclusion (36) is equivalent to the attainability condition (34) in Assumption 6, namely

$$
\mathcal{A} \hat{B} f(\rho) \supset-\mathcal{Q} \hat{B} f(\rho)+f(\rho) \quad \Leftrightarrow \quad \operatorname{val}[\lambda]<0, \forall \lambda \in \mathbb{R}^{m} .
$$

Proof: If condition (36) holds, then for all $\lambda \in \mathbb{R}^{m}$,

$$
\exists \alpha \in \mathcal{U} \mid \quad \lambda^{T} A(\alpha) \hat{B} f(\rho)<\min _{\omega \in \mathcal{W}} \lambda^{T}(-Q(\omega) \hat{B} f(\rho)+f(\rho)) .
$$

Recalling that any point in a convex set can be expressed as a convex combination of its vertices, we have $\alpha=\sum_{i=1}^{p} a_{k} a^{(k)}$ and similarly $\omega=\sum_{k=1}^{q} w_{k} w^{(k)}$. The above condition then 
corresponds to saying that for all $\lambda \in \mathbb{R}^{m}$ there exists $\alpha \in \mathcal{U}$ such that

$$
\begin{aligned}
& \lambda^{T} A\left(\sum_{i=1}^{p} a_{k} a^{(k)}\right) \hat{B} f(\rho) \\
& <\min _{\omega \in \mathcal{W}} \lambda^{T}\left(-Q\left(\sum_{k=1}^{q} w_{k} w^{(k)}\right) \hat{B} f(\rho)+f(\rho)\right) .
\end{aligned}
$$

From the above condition, we can derive equivalently that for all $\lambda \in \mathbb{R}^{m}$ there exists $\alpha \in \mathcal{U}$ such that for all $w \in \mathcal{W}$

$$
\lambda^{T}\left(A\left(\sum_{i=1}^{p} a_{k} a^{(k)}\right) \hat{B} f(\rho)+Q\left(\sum_{k=1}^{q} w_{k} w^{(k)}\right) \hat{B} f(\rho)-f(\rho)\right)<0 .
$$

The above condition can be rewritten as, for all $\lambda \in \mathbb{R}^{m}$

$$
\exists \alpha \in \mathcal{U} \mid \quad \lambda^{T}\left(\tilde{B}^{T}(\alpha, \omega) \hat{B}-I\right) f(\rho)<0, \forall \omega \in \mathcal{W} .
$$

Recalling the definition $D_{1}(\alpha)$ $\left\{\left(\tilde{B}^{T}(\alpha, \omega) \hat{B}-I\right) f(\rho): \omega \in \mathcal{W}\right\}$, the above condition implies that

$$
\exists \alpha \in \mathcal{U} \mid \quad D_{1}(\alpha) \subseteq H=\left\{\hat{\rho} \in \mathbb{R}^{m} \mid \lambda^{T} \hat{\rho}<0\right\} .
$$

We conclude our proof by taking $\lambda=\rho-y$ where $y \in \Pi_{\mathcal{M}}(\rho)$ and observing that this coincides with the attainability condition (34).

So far, we cannot say much about the speed of convergence of the density vector to the pre-assigned set. Under stronger conditions, we can have exponential convergence as established next.

Assumption 7: (Exponential attainability of set $\mathcal{M}$ ) Let $\mathcal{M}$ be given as in (4), $r>0$ and $U=\left\{\rho \in \mathbb{R}^{m}\right.$ : $\operatorname{dist}(\rho, \mathcal{M})<r\}$. For all $\rho \in U \backslash \mathcal{M}$ there exists $y \in \Pi_{\mathcal{M}}(\rho)$ such that the value of the projected game, val $[\lambda]$, is upper bounded by $-\lambda^{T} \lambda$, for every $\lambda=\rho-y$, i.e.,

$$
\begin{aligned}
\operatorname{val}[\lambda]:= & \inf _{\alpha} \sup _{\omega}\left\{\lambda^{T}\right. \\
& \left.\cdot\left(\tilde{B}^{T}(\alpha, \omega) \hat{B}-I\right) f(\rho)\right\}<-\lambda^{T} \lambda, \quad \forall \lambda=\rho-y .
\end{aligned}
$$

Theorem 6: Let Assumption 7 hold true. Then, dynamics (23) converges exponentially to $\mathcal{M}$, namely

$$
\operatorname{dist}(\rho(t), \mathcal{M})=e^{-t} \operatorname{dist}(\rho(0), \mathcal{M}) .
$$

Proof: Let $\rho$ be a solution of dynamics (23) with initial value $\rho(0) \in U \backslash \mathcal{M}$. Set $\tau=\{\inf t>0 \mid \rho(t) \in \mathcal{M}\} \leq \infty$ and let $V(\rho(t))=\operatorname{dist}(\rho(t), \mathcal{M})$. For all $t \in[0, \tau]$ and $y \in$ $\Pi_{\mathcal{M}}(\rho(t))$

$$
\begin{aligned}
& V(\rho(t+d t))-V(\rho(t))=\|\rho(t+d t)-y\|-\|\rho(t)-y\| \\
& =\|\rho(t)+\dot{\rho}(t) d t-y\|-\|\rho(t)-y\|+|d t| \epsilon(d t) \\
& =\frac{1}{\|\rho(t)+\dot{\rho}(t) d t-y\|}\|\rho(t)+\dot{\rho}(t) d t-y\|^{2} \\
& -\frac{1}{\|\rho(t)-y\|}\|\rho(t)-y\|^{2}+|d t| \epsilon(d t)
\end{aligned}
$$

where $\lim _{d t \rightarrow 0} \epsilon(d t)=0$. Hence

$$
\begin{aligned}
\dot{V}(\rho(t)) & =\lim _{d t \rightarrow 0} \frac{1}{d t}\left(\frac{1}{\|\rho(t)+\dot{\rho}(t) d t-y\|}\|\rho(t)+\dot{\rho}(t) d t-y\|^{2}\right. \\
& \left.-\frac{1}{\|\rho(t)-y\|}\|\rho(t)-y\|^{2}+|d t| \epsilon(d t) \mid\right) \\
& =\frac{1}{\|\rho(t)-y\|} \lim _{d t \rightarrow 0} \frac{1}{d t}\left(\|\rho(t)+\dot{\rho}(t) d t-y\|^{2}\right. \\
& \left.-\|\rho(t)-y\|^{2}\right) \\
& =\frac{1}{\|\rho(t)-y\|} \frac{d}{d t}\left(\|\rho(t)-y\|^{2}\right) \\
& \leq \frac{2}{\|\rho(t)-y\|}(\rho(t)-y)^{T} \dot{\rho}(t) .
\end{aligned}
$$

Now, as $\mathcal{M}$ is a compact set, from Assumption 7 we have that for all $\rho \in U \backslash \mathcal{M}$ there exists $y \in \Pi_{\mathcal{M}}(\rho)$ such that the affine hyperplane orthogonal to $[\rho(t), y]$ at $y$ separates $\rho(t)-y$ from $\dot{\rho}(t)$, namely

$$
\begin{aligned}
& \operatorname{val}[\rho(t)-y]:=\inf _{\alpha} \sup _{\omega}\left\{(\rho(t)-y)^{T}\right. \\
& \left.\quad \cdot\left(\tilde{B}^{T}(\alpha, \omega) \hat{B}-I\right) f(\rho)\right\} \\
& =\inf _{\alpha} \sup _{\omega}\left\{(\rho(t)-y)^{T} \dot{\rho}(t)\right\}<-(\rho(t)-y)^{T}(\rho(t)-y)
\end{aligned}
$$

from which we have

$$
\begin{aligned}
& \dot{V}(\rho(t)) \leq \frac{2}{\|\rho(t)-y\|}(\rho(t)-y)^{T} \dot{\rho}(t) \\
& =-2 V(\rho(t))+\frac{2}{\|\rho(t)-y\|}(\rho(t)-y)^{T}(\rho(t)-y+\dot{\rho}(t)),
\end{aligned}
$$

and this concludes our proof.

The above result is in the same spirit of convergence conditions in set inclusion theory discussed in [10], [11].

\section{NUMERICAL EXAMPLE}

Consider the following example, consisting of 4 vertices and 5 edges, as shown in Fig. 4 (vertex ' $S$ ' stands for the source and vertex ' $\mathrm{D}$ ' stands for the destination, edge $e$ is marked with $f_{e}$, the incoming flow $f_{0}$ is equal to the outgoing flow $\left.f_{6}=f_{4}+f_{5}\right)$.

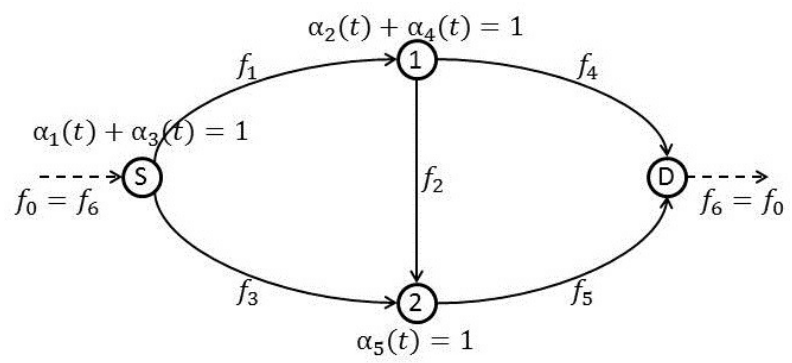

Fig. 4: Network system.

The matrices introduced in the sections above are

$$
\begin{gathered}
\tilde{B}^{T}(\alpha)=\left[\begin{array}{ccc}
\alpha_{1} & 0 & 0 \\
0 & \alpha_{2} & 0 \\
\alpha_{3} & 0 & 0 \\
0 & \alpha_{4} & 0 \\
0 & 0 & \alpha_{5}
\end{array}\right] \\
\hat{B}=\left[\begin{array}{lllll}
0 & 0 & 0 & 1 & 1 \\
1 & 0 & 0 & 0 & 0 \\
0 & 1 & 1 & 0 & 0
\end{array}\right]
\end{gathered}
$$

The density evolution expressed by (2) takes on the form, where we use $f_{e}\left(\rho_{e}(t)\right)=\phi \rho_{e}(t)$ :

$$
\left\{\begin{array}{l}
\dot{\rho}_{1}(t)=\alpha_{1}(t)\left(\phi \rho_{4}(t)+\phi \rho_{5}(t)\right)-\phi \rho_{1}(t) \\
\dot{\rho}_{2}(t)=\alpha_{2}(t) \phi \rho_{1}(t)-\phi \rho_{2}(t) \\
\dot{\rho}_{3}(t)=\alpha_{3}(t)\left(\phi \rho_{4}(t)+\phi \rho_{5}(t)\right)-\phi \rho_{3}(t) \\
\dot{\rho}_{4}(t)=\alpha_{4}(t) \phi \rho_{1}(t)-\phi \rho_{4}(t) \\
\dot{\rho}_{5}(t)=\alpha_{5}(t)\left(\phi \rho_{2}(t)+\phi \rho_{3}(t)\right)-\phi \rho_{5}(t)
\end{array}\right.
$$

and

$$
\begin{cases}\alpha_{1}(t)+\alpha_{3}(t) & =1 \\ \alpha_{2}(t)+\alpha_{4}(t) & =1 \\ \alpha_{5}(t) & =1\end{cases}
$$




\begin{tabular}{|c|c|c|c|}
\hline Parameter & Value & Variable & Initial Value \\
\hline$\phi$ & 0.8 & $\rho(t)$ & $(0.3,0.5,0.2,0,0)$ \\
\hline Time step $h$ & 0.01 & $\alpha(t)$ & $(0.6,0.5,0.4,0.5,1)$ \\
\hline Time span $T$ & 20 & & \\
\hline
\end{tabular}

TABLE III: Parameters of the overall system.

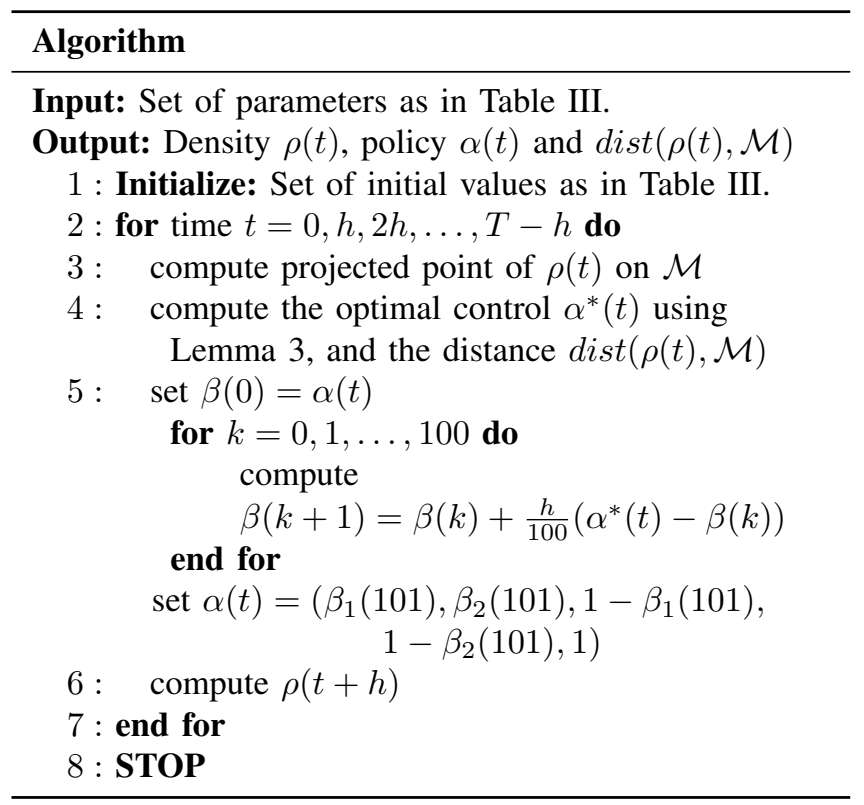

Let us consider the paths $\{1,4\},\{1,2,5\}$ and $\{3,5\}$. In other words, $\mathcal{P}=\{\{1,4\},\{1,2,5\},\{3,5\}\}$ which corresponds to defining an output

$$
\left[\begin{array}{l}
y_{1}(t) \\
y_{2}(t) \\
y_{3}(t)
\end{array}\right]=\underbrace{\left[\begin{array}{lllll}
1 & 0 & 0 & 1 & 0 \\
1 & 1 & 0 & 0 & 1 \\
0 & 0 & 1 & 0 & 1
\end{array}\right]}_{C}\left[\begin{array}{c}
\rho_{1}(t) \\
\rho_{2}(t) \\
\rho_{3}(t) \\
\rho_{4}(t) \\
\rho_{5}(t)
\end{array}\right]
$$

\section{A. Deterministic case}

We first consider the deterministic case. Table III shows the parameters of the overall system. According to Lemma 3, we have the following Algorithm to solve the distributed routing problem. The simulations are carried out with MATLAB on an Inter(R) Xeon(R) CPU E31245 at 3.30GHz and 8 GB of RAM, and the results are illustrated in Figures 5-7. The run time of the simulation is around 25 seconds. Since $\sum_{e} \dot{\rho}_{e}(t)=0$ (i.e., conservation law holds), $\sum_{e} \rho_{e}(t)=\sum_{e} \rho_{e}(0)=1$ always holds, which is shown in Fig. 5. When achieving consensus, $\rho_{2}(t)=0$ holds, indicating that all players choose either leaving the source vertex through edge 1 and returning it through edge 4 , or leaving through edge 3 and going back through edge 5 . Moreover, the players choose these two routes almost equiprobably, i.e., $\alpha_{1} \approx \alpha_{3} \approx 0.5$, as illustrated in Fig. 6 . The distance from the consensus set converges to zero, as illustrated in Fig. 7. Note that in order to avoid chirping in $\alpha(t)$, we have introduced lowpass dynamics $\dot{\beta}(t)=\alpha^{*}(t)-\beta(t)$ (the relevant transfer function is $\beta(s)=\frac{1}{s+1} \alpha^{*}(s)$ which is

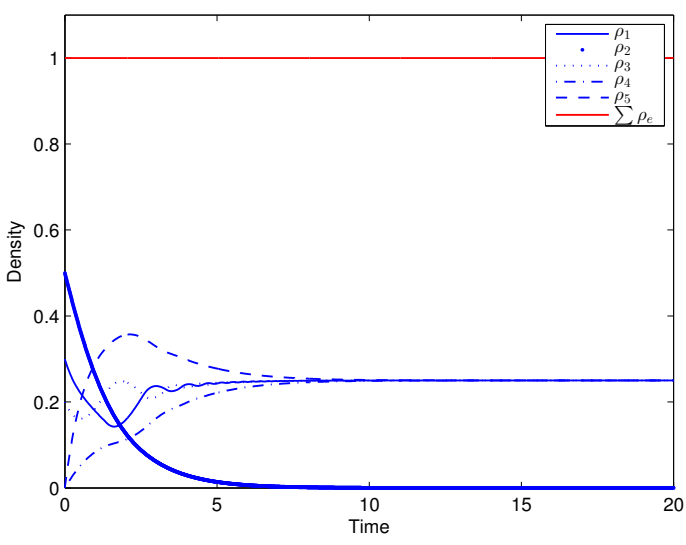

Fig. 5: Simulation results of the deterministic case: density.

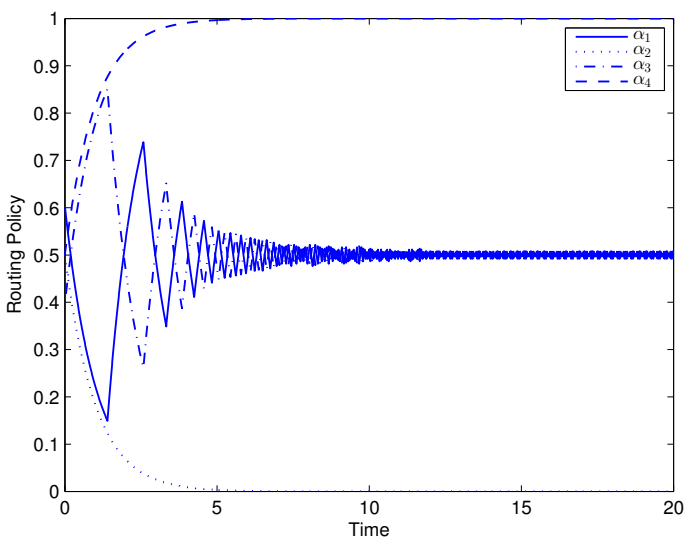

Fig. 6: Simulation results of the deterministic case: routing policy $\left(\alpha_{5}(t)=1\right.$ holds all the time).

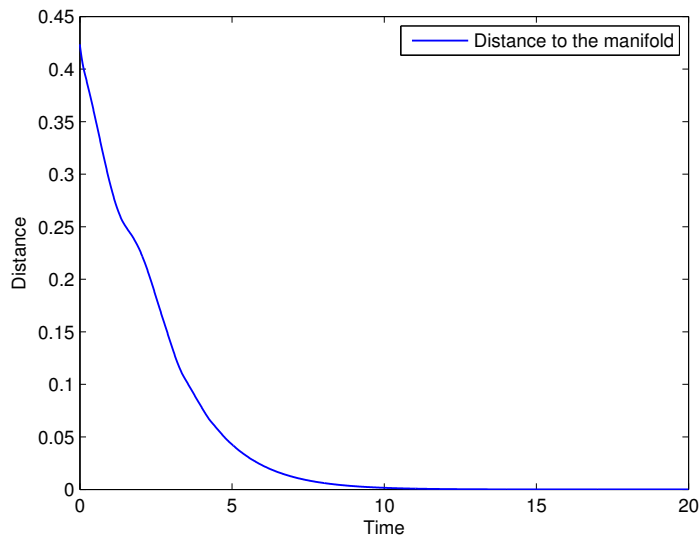

Fig. 7: Simulation results of the deterministic case: distance to the consensus set.

actually a lowpass filter for $\alpha(t)$ ), corresponding to Step 5 in the Algorithm. 


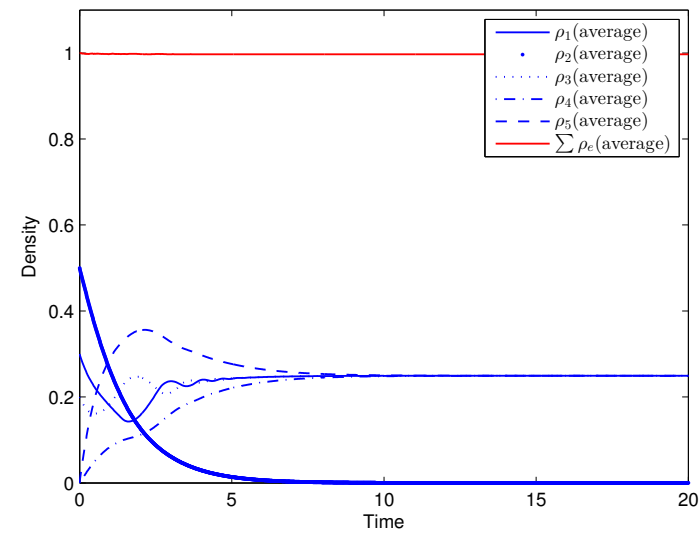

Fig. 8: Simulation results of the stochastic case: average density.

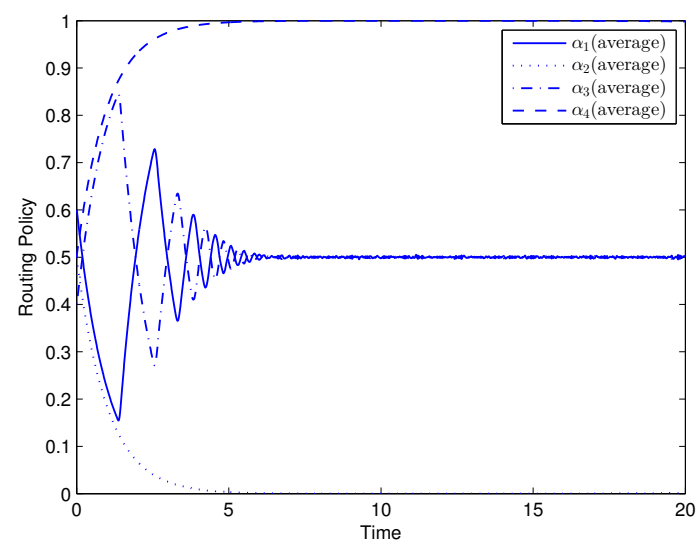

Fig. 9: Simulation results of the stochastic case: average routing policy $\left(\alpha_{5}(t)=1\right.$ holds all the time).

\section{B. Stochastic case}

We now consider the stochastic case. In this case, the dynamics of the network (37) change to

$$
\left\{\begin{array}{l}
\dot{\rho}_{1}(t)=\alpha_{1}(t)\left(\phi \rho_{4}(t)+\phi \rho_{5}(t)\right)-\phi \rho_{1}(t)+w_{1}(t) \\
\dot{\rho}_{2}(t)=\alpha_{2}(t) \phi \rho_{1}(t)-\phi \rho_{2}(t)+w_{2}(t) \\
\dot{\rho}_{3}(t)=\alpha_{3}(t)\left(\phi \rho_{4}(t)+\phi \rho_{5}(t)\right)-\phi \rho_{3}(t)+w_{3}(t) \\
\dot{\rho}_{4}(t)=\alpha_{4}(t) \phi \rho_{1}(t)-\phi \rho_{4}(t)+w_{4}(t) \\
\dot{\rho}_{5}(t)=\alpha_{5}(t)\left(\phi \rho_{2}(t)+\phi \rho_{3}(t)\right)-\phi \rho_{5}(t)+w_{5}(t)
\end{array}\right.
$$

where $w_{e}(t)$ represents the Gaussian noise whose mean is 0 and variance is $\frac{1}{2} \sigma^{2} \operatorname{dist}^{2}(\rho(t), \mathcal{M})$. The above algorithm can still solve the distributed routing problem. We continue to use the parameters in Table III, set $\sigma=1$, run 50 different Monte Carlo trajectories, and compute the average value of these trajectories (see Figures 8-10). We can see that the average trajectories are almost the same as those in the deterministic case. Moreover, the average trajectory of $\alpha(t)$ is now much more smooth. The sampled average distance from the consensus set converges to zero.

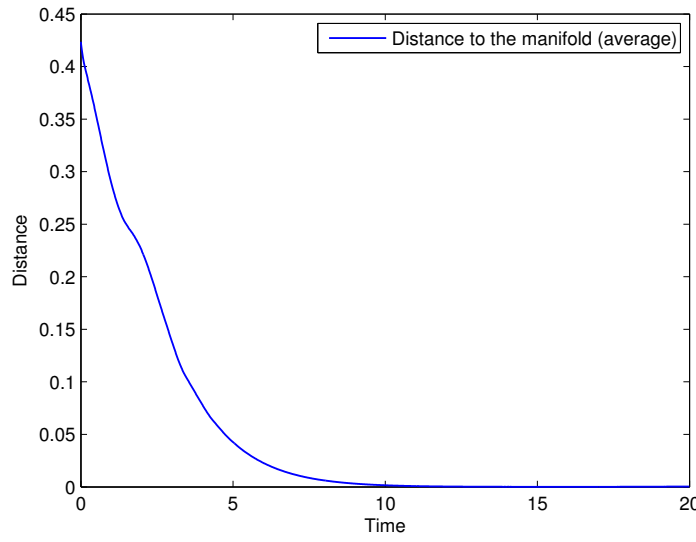

Fig. 10: Simulation results of the stochastic case: average distance to the consensus set.

\section{CONCLUSIONS AND FUtURE DiRECTIONS}

In this paper, we have provided a mean-field game formulation of a distributed routing problem, which intersects recent research on optimal planning and transportation. The problem setup has been motivated by an idea in [14], [15] that develops a dynamical model for the density at network edges in a locally responsive traffic network. This paper has provided several contributions. Beyond the mean-field game formulation, we have illustrated an extended state space solution approach applied to both stochastic and worst-case scenarios. In both cases, the study has analyzed convergence conditions of the density to a pre-assigned set. Connections with repeated games with vector payoffs and set inclusion theory have been highlighted.

We wish to extend the study in at least three different directions. Still within the realm of robust networks, alternative models of adversarial disturbances can be imported in the problem at hand, as for instance energy bounded disturbances in the spirit of $H_{\infty}$ optimal control. Regarding the network topology, our conjecture is that more can be said in terms of speed of convergence and properties of the underlying graph such as graph connectivity, degree of nodes, eigenvalues of graph Laplacian matrices. In this context, special attention will be given to specific graph structures. A third direction involves the analysis of non-conservative flows. Actually, the present analysis assumes that the population does not grow or diminishes over time. A more complex scenario would consider the case where the number of players changes with time, perhaps dependently on the level of congestion in the network. These topics are left for future research.

\section{REFERENCES}

[1] Y. Achdou, F. Camilli, and I. Capuzzo Dolcetta, "Mean field games: numerical methods for the planning problem", SIAM Journal of Control and Optimization, vol. 50, 2012, pp. 77-109.

[2] F. Bagagiolo and D. Bauso, "Mean-field games and dynamic demand management in power grids", Dynamic Games and Applications, vol. 4, 2014, pp. $155-176$.

[3] M. Bardi, "Explicit solutions of some Linear-Quadratic Mean Field Games", Network and Heterogeneous Media, vol. 7, 2012, pp. 243-261. 
[4] D. Bauso, Game Theory with Engineering Applications, SIAM's Advances in Design and Control series, Philadelphia, PA, USA, 2016.

[5] D. Bauso, F. Blanchini, and R. Pesenti, "Optimization of Long-run Average-flow Cost in Networks with time-varying unknown demand", IEEE Transactions on Automatic Control, vol. 55, no.1, 2010, pp. 20-31.

[6] D. Bauso, E. Solan, E. Lehrer, and X. Venel, "Attainability in Repeated Games with Vector Payoffs", INFORMS Mathematics of Operations Research, in print. Available on Arxiv at http://arxiv.org/abs/1201.6054.

[7] D. Bauso, T. Mylvaganam, and A. Astolfi, "Approximate solutions for crowd-averse robust mean-field games", Proceedings of the 13th European Control Conference, Strasbourg, 2014, pp. 1217-1222.

[8] D. Bauso, X. Zhang, and A. Papachristodoulou, "Density Flow over Networks: A Mean-Field Game Theoretic Approach", Proceedings of the 53rd IEEE Conference on Decision and Control, Los Angeles, USA, 2014.

[9] J.-D. Benamou and Y. Brenier, "A computational fluid mechanics solution to the Monge-Kantorovich mass transfer problem", Numerische Mathematik, vol. 84, no. 3, 2000, pp. 375-393.

[10] M. Benaïm, J. Hofbauer, and S. Sorin, "Stochastic Approximations and Differential Inclusions", SIAM Journal on Control and Optimization, vol. 44, no. 1, 2005, pp. 328-348.

[11] M. Benaïm, J. Hofbauer, and S. Sorin, "Stochastic Approximations and Differential Inclusions, Part II: Applications", INFORMS Mathematics of Operations Research, vol. 31, no. 4, 2006, pp. 673-695.

[12] F. Blanchini, S. Miani, and W. Ukovich, "Control of productiondistribution systems with unknown inputs and system failures", IEEE Transactions on Automatic Control, vol. 45, no. 6, 2000, pp. 1072-1081.

[13] F. Blanchini, F. Rinaldi, and W. Ukovich, "Least inventory control of multi-storage systems with non-stochastic unknown input", IEEE Transactions on Robotics and Automation, vol. 13, 1997, pp. 633-645.

[14] G. Como, K. Savla, D. Acemoglu, M. Dahleh, and E. Frazzoli, "Distributed robust routing in dynamical networks - Part II: strong resilience, equilibrium selection and cascaded failures", IEEE Transactions on Automatic Control, vol. 58, no. 2, 2013, pp. 333-348.

[15] G. Como, K. Savla, D. Acemoglu, M. Dahleh, and E. Frazzoli, "Distributed robust routing in dynamical networks - Part I: Locally responsive policies and weak resilience", IEEE Transactions on Automatic Control, vol. 58, no. 2, 2013, pp. 317-332.

[16] D. A. Gomes, J. Mohr, R. R. Souza, "Discrete time, finite state space mean field games", J. Math. Pures Appl, vol. 93, no. 3, 2010, pp. 308328.

[17] D. A. Gomes, J. Mohr, R. R. Souza, "Continuous time finite state mean field games", Appl. Math. Optim, vol. 68, no. 1, 2013, 99-143.

[18] O. Gueant, J. M. Lasry, and P. L. Lions, "Mean field games and applications", Paris-Princeton Lectures, Springer, 2010, pp. 1-66.

[19] M.Y. Huang, P.E. Caines, and R.P. Malhamé, "Individual and Mass Behaviour in Large Population Stochastic Wireless Power Control Problems: Centralized and Nash Equilibrium Solutions", IEEE Conference on Decision and Control, HI, USA, 2003, pp. 98-103.

[20] M.Y. Huang, P.E. Caines, and R.P. Malhamé, "Large Population Stochastic Dynamic Games: Closed Loop Kean-Vlasov Systems and the Nash Certainty Equivalence Principle", Communications in Information and Systems, vol. 6, no. 3, 2006, pp. 221-252.

[21] M.Y. Huang, P.E. Caines, and R.P. Malhamé, "Large population costcoupled LQG problems with non-uniform agents: individual-mass behaviour and decentralized $\epsilon$-Nash equilibria", IEEE Transactions on Automatic Control, vol. 52. no. 9, 2007, pp. 1560-1571.

[22] B. Jovanovic, and R. W. Rosenthal, "Anonymous sequential games", Journal of Mathematical Economics, vol. 17, 1988, pp. 77-87.

[23] J.-M. Lasry and P.-L. Lions, "Jeux à champ moyen. I Le cas stationnaire", Comptes Rendus Mathematique, vol. 343, no. 9, 2006, pp. 619625 .

[24] J.-M. Lasry and P.-L. Lions, "Jeux à champ moyen. II Horizon fini et controle optimal". Comptes Rendus Mathematique, vol. 343, no. 10, 2006, pp. 679-684.

[25] J.-M. Lasry and P.-L. Lions, "Mean field games", Japanese Journal of Mathematics, vol. 2, 2007, pp. 229-260.

[26] E. Lehrer, E. Solan, D. Bauso, "Repeated games over networks with vector payoffs: the notion of attainability", Proceedings of the Int. Conf. on NETwork Games, COntrol and OPtimization (NetGCooP 2011) Paris, 2011.

[27] P.-L. Lions, Cours au Collège de France, www.college-de-france.fr.

[28] R. Pesenti, and D. Bauso, "Mean field linear quadratic games with set up costs", Dynamic Games and Applications, vol. 3, no. 1, 2013, pp. 89-104.
[29] A. Porretta, "On the Planning Problem for the Mean Field Games System", Dynamic Games and Applications, April 2013, available online, dpi:10.1007/s13235-013-0080-0

[30] H. Tembine, Mean field stochastic games, Notes (2011).

[31] H. Tembine, Q. Zhu, and T. Başar, "Risk-sensitive mean-field stochastic differential games", Proceedings of 2011 IFAC World Congress, Milan, Italy, August 29 - September 2, 2011.

[32] H. Tembine, Q. Zhu, T. Başar, "Risk-sensitive mean-field games", IEEE Transactions on Automatic Control, vol. 59, no. 4, 2014, pp. 835-850.

[33] H. Tembine, D. Bauso, and T. Basar, "Robust linear quadratic mean-field games in crowd-seeking social networks", Proceedings of the 52nd IEEE Conference on Decision and Control, Florence, IT, 2013, pp. 3134-3139.

[34] G. Y. Weintraub, C. Benkard, and B. Van Roy, "Oblivious Equilibrium: A Mean Field Approximation for Large-Scale Dynamic Games", Advances in Neural Information Processing Systems, MIT Press, 2005.

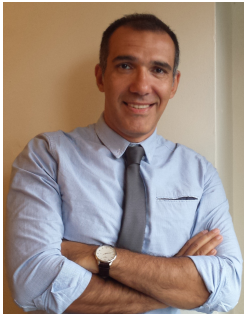

Dario Bauso received his Laurea degree in Aeronautical Engineering in 2000 and his Ph.D. degree in Automatic Control and Systems Theory in 2004 from the University of Palermo, Italy. Since 2015 he has been with the Department of Automatic Control and Systems Engineering, The University of Sheffield (UK), where he is currently Reader in Control and Systems Engineering. Since 2005 he has also been with the Dipartimento di Ingegneria Chimica, Gestionale, Informatica, Meccanica, University of Palermo (Italy), where he is currently Associate Professor of Operations Research. His research interests are in the field of Optimization, Optimal and Distributed Control, and Game Theory. Since 2010 he is member of the Conference Editorial Board of the IEEE Control Systems Society. He is Associate Editor of IEEE Transactions on Automatic Control, Automatica, and Dynamic Games and Applications. He is the author of the book Game Theory with Engineering Applications, edited by SIAM in 2016.

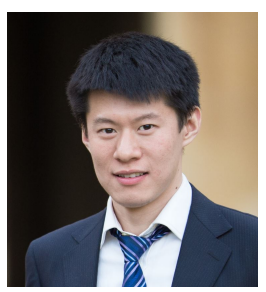

Xuan Zhang obtained the B.Eng. degree in Control Engineering from Tsinghua University, Beijing, China, in 2011. In 2015, he completed his Ph.D. programme in Department of Engineering Science at the University of Oxford, UK. He is now a Post Doctoral Fellow in both the School of Engineering and Applied Sciences, and Harvard Center for Green Buildings and Cities, at Harvard University, USA. His research interests include the control and optimization for smart power networks, network congestion control, consensus of multi-agent systems, and the stability and robustness analysis of nonlinear dynamical systems.

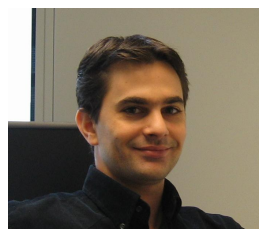

Antonis Papachristodoulou received the M.A./M.Eng. degree in electrical and information sciences from the University of Cambridge, Cambridge, U.K., and the Ph.D. degree in control and dynamical systems (with a minor in aeronautics) from the California Institute of Technology, Pasadena, CA, USA. Currently, he is an Associate Professor in Engineering Science at the University of Oxford, Oxford, U.K., and a Tutorial Fellow at Worcester College, Oxford, U.K. He is also an EPSRC Fellow for Growth in Synthetic Biology and Director of the EPSRC \& BBSRC Centre for Doctoral Training in Synthetic Biology. His research interests include large-scale nonlinear systems analysis, sum of squares programming, synthetic and systems biology, networked systems, and flow control. Prof. Papachristodoulou received the 2015 European Control Award for his contributions to robustness analysis and applications to networked control systems and systems biology. In the same year, he received the O. Hugo Schuck Best Paper Award. 